\title{
A Novel Method of Probing the Fractional Composition of Nanosystems ${ }^{\dagger}$
}

\author{
Kozlov A.S. ${ }^{1 *}$, Petrov A.K. ${ }^{1}$, Kulipanov G.N. ${ }^{2}$, Aseyev V. ${ }^{3}$ \\ Institute of chemical kinetics and combustion $S B R A S^{1}$, \\ G.I. Budker Institute of nuclear physics $S B R A S^{2}$, \\ University of Helsinki ${ }^{3}$
}

\begin{abstract}
The article presents a comparison of the traditional methods to investigate the fractional composition of nanosized powders, namely atomic force microscopy (AFM), scanning electron microscopy (SEM), dynamic light scatterin, (DLS) and a new one - analysis of the aerosol products of submillimeter pulse laser ablation (SLA). As has previously been proven, biological macromolecules retain activity after ablation under submillimeter wavelengths, molecules become separated in the aerosol phase and each sort of molecues forms its own fraction of the aerosol particles. We suggest that this process is made possible as the result of influence on the hydrogen and van der Waals' bonds, with the energies within the submillimeter range. Results of investigations on $\mathrm{SiO}_{2}$ and artificial diamond clusters using the above methods in both powder and colloid states are discussed. SLA with subsequent detection of aerosol products with the help of convenient aerosol equipment is found to be simple, fast and informative, and can act as a competitor to mass spectrometry, X-ray scattering and other methods.
\end{abstract}

Keywords: THz laser ablation, nanopowders, aerosol analysis

\section{Introduction}

Laser ablation is a phenomenon thas is sure to gain broad application in science, technology ${ }^{1)}$ and medicine ${ }^{2)}$. At present, the case in point is the use of the radiation with wavelengths shorter than $10 \mu \mathrm{m}$, and this implies the destruction of a material at the molecular level. Use of the submillimeter radiation opens novel outlooks in this area. The submillimeter (terahertz) radiation is the region of the electromagnetic spectrum between the far infrared and microwave radiation. Usually this region corresponds to wavelengths from 100 to $500 \mu \mathrm{m}$. This region has become available to researchers due to the recent development of new powerful sources of $\mathrm{THz}$ radiation. One of these sources is the Free Electron Laser (FEL), developed and built at the Institute of Nuclear

\footnotetext{
Accepted: September $23^{\text {rd }}, 2010$

630090, Institutskaya 3, Novosibirsk, Russia

2 630090, Lavrentiev prospect 11, Novosibirsk, Russia

3 PB 55, AI Virtasen Aukio 1, FIN-00014 HY, Helsinki, Finland

* Corresponding author

E-mail: kozlov@kinetics.nsc.ru, TEL.: +73833332944, FAX: +73833307350
}

Physics SB RAS. The radiation of this laser is pulsed wite a pulse duration of about 50 picoseconds ane a pulse period of 180 nanoseconds; the laser wavelength is tunable within the range 120 to 240 micrometers with the relative radiation line width about $10^{-3}$, average power up to $400 \mathrm{~W}$, and peak power up to 0.8 $\mathrm{MW}^{3)}$.

After the soft nondestructive ablation of biological macromolecules was discovered ${ }^{4,5}$, the possibility arose to develop a principally new method to measure the size of nanoparticles and nanosystems of all kinds.

The phenomenon of soft nondestructive ablation involves the transport of macromolecules from a solid substrate or from solution into the aerosol phase under the action of laser radiatiof in the terahertz range; the structure and initial properties of the macromolecules aredretained. A specific feature of this radiation is small quantum energy, $\sim 0.01 \mathrm{eV}$, which is comparable with the energy of the hydrogen and van der Waals' bonds but much less than the energy of the covalent bonds. So, the terahertz radiation is unable to dissociate molecules with a single quantum, which allowg the molecules to be transferred into the 
aerosor phase without destroying the primary structore Experiments were carried out with lyophilizates and colloids of DNA, proteins and enzymes. The process of transition into the aerosol phase occurs without destruction and denaturation of molecules; all their biological properties aredpreserved. In this process, an individual fraction of macromolecules forms an individual fraction of the aerosol particles. Binary and ternary mixtures form two or three fractions of the aerosol particles, respectively ${ }^{4,5)}$. The molecular masses of these fractions correspond to the molecular masses of the initial components. This circumstance suggests that a new universal method may be developed to determine the size of nanoparticles of any origin.

Determination of the fraction composition of nanopowders, nanocolloids and biological macromolecules is of significant interest for academic and for practical applications. Conventional methods used for this purpose are X-ray scattering, electron microscopy, and mass spectrometry; these methods are timeconsuming, expensive and sometimes ambiguous. We propose to use terahertz laser ablation for the nondestructive transfer of a nanodispersed system into the gas phase, followed by its analysis using conventional aerosol instruments. In the present work, we used an automatic diffusion battery with the condensation particle counter ${ }^{6)}$ to determine the particle size distribution of ablation products within the size range $3-250 \mathrm{~nm}$; the source of the terahertz radiation was the free electron laser (FEL) of the Siberian Center for Photochemical Research ${ }^{3)}$. Ultrafind diamond-carbon powder and ultrafine silicon dioxide powder were used as the subject of investigation.

To demonstrate the capability of the proposed method, the results of the sample investigation obtained by means of the soft laser ablation were compared with the results obtained using conventional methods: dynamic light scattering (photon correlation spectroscopy), scanning electron and atomic force microscopy at the Department of Chemistry of the University of Helsinki.

\section{Experimental technology and sample treatment}

Commercially available ultrafind diamond-carbon and $\mathrm{SiO}_{2}$ powders were used as the samples. The ultrafind diamond-carbon powder was obtained by detonating solid explosives at FR\&PC (ALTAI) (Biysk, Russia). As reported by the manufacturer, the powder contains 40-60 mass per cent of ultrafine diamond. Particle size is $5-20 \mathrm{~nm}$. The ultrafine $\mathrm{SiO}_{2}$ powder "Tarkosil" was manufactured at the INP SB RAS by means of electron impart. According to the manufacturer's data, the average size of powder particles is $18-25 \mathrm{~nm}$.

To determine the particle size distribution of the powders with conventional methods, we used the scanning electron microscope Hitachi S-4800, atomic force microscope Veeco Instrument Nanoscope V (tapping mode). Light scattering measurements were conducted using a goniometer BI-200SM, two BI-DS1 detectors, and a digital cross-correlator TurboCorr (Brookhaven Instrument Corporation). Methodological aspects of the method can be found elsewhere ${ }^{7}$. Time-correlation functions were analyzed with an inverse Laplace transform program CONTIN (BIC software). The mean peak value of a size distribution was a matter of choice to estimate an apparent hydrodynamic radius of scattering objects.

Intense sample preparation was carried out before the DLS and microscopic examination. The samples were transferred into a diluted solution wite a concentration of $0.01-1 \mathrm{mg} / \mathrm{ml}$, and sodium dodecyl sulfate, SDS, was added to provide colloidal stability of particles. To disintegrate the interparticle aggregates, an intense ultrasonic treatment of the solution was carried out, followed by filtration through a membrane with pore diameter $0.45 \mu \mathrm{m}$. Measurements of the light scattering signal were carried out at the wavelength of $488 \mathrm{~nm}$ at angles $90^{\circ}$ and $140^{\circ}$ with serial dilutions of the solution under investigation. In addition, for the atomic force and scanning electron microscopy studies, the prepared solutions were additionally subjected te lyophilic drying directly on the microscopic substrates. To be sure in the absence of any significant effect the sample preparation procedure, we studied the samples at all stages of this procedure. An example of the micrograph of the prepared sample of diamond-carbon powder, obtained with the scanning electron microscope, is shown in Fig. 1. All the experiments were carried out at room temperature.

To study the samples by means of the submillimeter laser ablation, the sample was placed into a horizontal cell (Fig.2) into which an excess of filtered gaseous nitrogen was admitted, which was necessary to prevent thl ingress of aerosol from outside. Before starting the work, we always tested for the absence of aerosol in the entire air line. The power density necessary for the start of ablation was established by moving the sample along the focal axis of the sector mirror with the focal distance $\mathrm{f}=10 \mathrm{~cm}$; its average value was $20 \mathrm{~W} / \mathrm{cm}^{2}$. No special sample preparation 


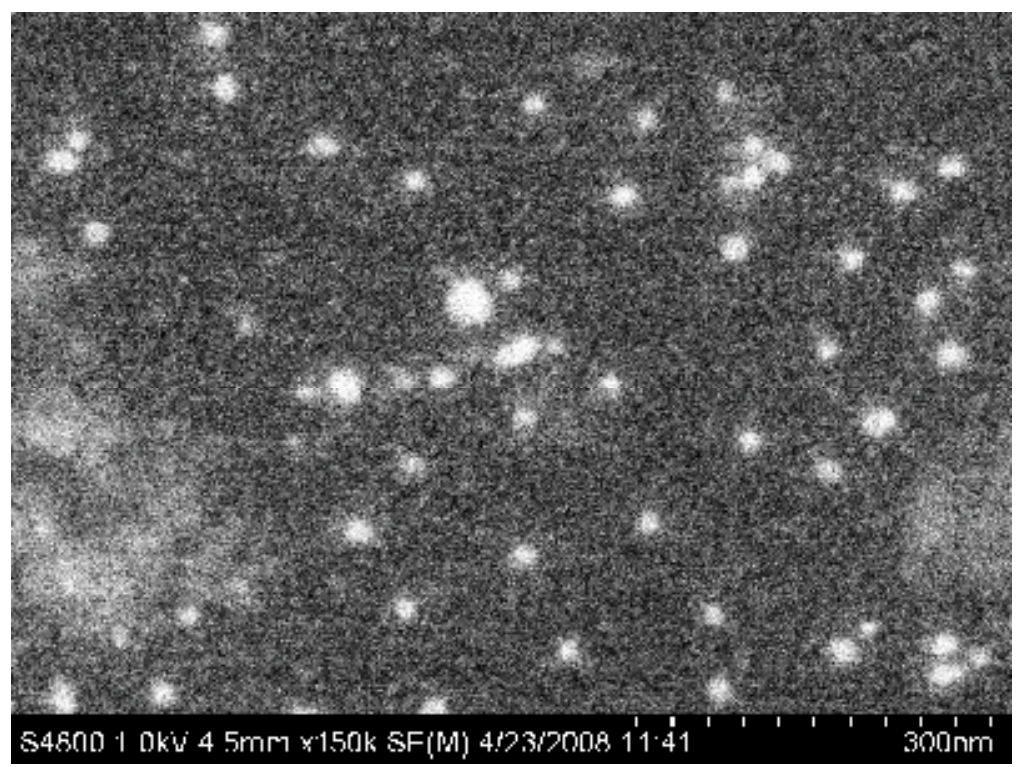

Fig. 1 An example of the micrograph of a sample of the prepared diamond-carbon powder, obtained with the scanning electron microscope.

was carried out before the ablation; the sample powders were placed on a substrate made of alumnium foil; deionized water was added ( $\sim 50 \mathrm{wt}$. per cent). Exposure was $3-10$ seconds. The resultant aerosol was conveyed with nitrogen flow into a buffer reservoir of 25 liters in volume to stabilize the number concentration of the particles. The aerosol was then brought to the diffusion spectrometer of aerosols (DSA) to analyze the particle size distributione The duration of a single measurement was 4 minutes. Measurements were carried out in series of 4 measurements per series, then the size distributions were averaged over the series. Irradiation of pure substrate did not cause the formation of particles. The total number of test on each sample was over 30 . The mean-root-square deviation of the average particle size did not exceed $15 \%$.
In the present work, aerosol particles were detected by means of the diffusion spectrometer of aerosols, which is intended to measure the particle size distribution and concentration of fine aerosols of natural and anthropogenic origin. The DSA may be used to solve a broad range of problems in atmospheric chemistry and physics, in environmental monitoring and the control of industry-related emissions, and also in laboratory aerosol research. The range of measurable particle diameters is 3 to 250 $\mathrm{nm}$. The range of particle concentrations measured without dilution is $<5 \times 10^{5} \mathrm{~cm}^{-3}$. The flow rate of aerosol under investigation is $11 / \mathrm{min}$.

The performance of this instrument is based on the dependence of the diffusion coefficient of nanoparticles on their size ${ }^{8}$.

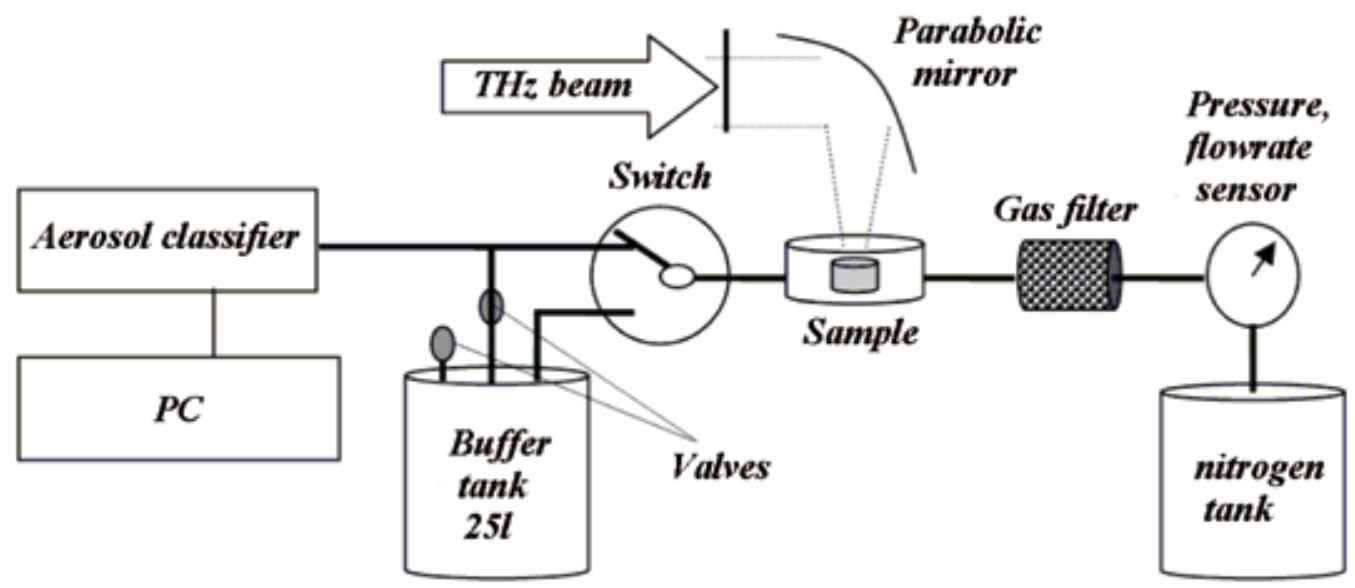

Fig. 2 Schematic of an experimental set-up. 
Here, $k$ is the Boltzmann constant, $T$ the temperature, $\eta$ the gas viscosity, $r$ the particle radius, $l$ the free path, $A=1.246, B=0.42, \mathrm{~b}=0.87$ are constants.

This dependence is the reason why the particles of different size have different deposition rates when an aerosol flow passes through porous media; in other words, smaller particles leave the flow faster, so that the coefficient of particle passage (penetration coefficient) through such a medium contains the information about the size of particles. This circumstance is used in diffusion spectrometers of aerosols.

The basic element of the DSA is a screen-type diffusion battery. The porous medium is a packet of screens placed perpendicular to the flow. The range of particle diameters measured with the DSA is 3 to $250 \mathrm{~nm}$. The scren -type battery is characterized by a simple dependence of the penetration coefficient K(r) on the particle size:

$$
K(r)=\left(1-B(D(r))^{\frac{2}{3}}\right)^{n}
$$

Here, $\mathrm{n}$ is the number of nets in the packet, $\mathrm{B}$ is a valug dependent on the design of the battery (the size and characteristics of the screens used in it) and on the gas flow rate through it. In the majority of cases, the second term in the bracketed expression is small, so (2) has the following form:

$$
K(r)=e^{-n B(D(r))^{\frac{2}{3}}}
$$

In the case of a polydisperse aerosol with the density of distribution $\varphi(r)$ and concentration $N$, according to (3), the concentration $N(n)$ at the outlet of the diffusion battery will be

$$
N(n)=N_{0} \int_{0}^{\infty} e^{-n B(D(r))^{\frac{2}{3}}} \varphi(r) d r
$$

One can see that the problem connected wite determination of the particle size distribution and concentration with the help of DSA is reduced to measuring $N(n)$ followed by solving the integral equation ${ }^{4}$.

So, measurement of the particle size distribution of the aerosol with the help of the diffusion battery involves consecutive counting of the concentration of aerosol particles as the aerosol flow passes through the cascades of screens (the number of cascades is 8 in our case); counting is performed with the help of the condensation counter of aerosol particles.

Diffusion batteries are a type of instruments that have made a good showing for the determination of the fractional composition of nanoaerosols during the last 50 years; they are produced by several manufacturers of research instrumentation (for example, TSI Inc., USA).

A number of natural restrictions arise when the instrument type under consideration is used. It follows from the equations presented above that only the radius of particles is taken into account during the recovery of distributions obtained with the diffusion battery, therefore, the particles whose shape differs from a sphere will be correlated, in the size distribution, with the size of a sphere having the same diffusion coefficient. Another limitation is connected with the measurement time. Indeed, the channels of the diffusion battery are scanned sequentially one after another. It is implied that both the number concentration of the aerosol and its particle size distribution remain unchanged during scanning. Special measures are taken in order to fulfill the latter condition. The total concentration of the aerosol under analysis is limited to $10^{4} \mathrm{~cm}^{-3}$, which makes us sure that the evolution of the distribution during measurement time is insignificant. This is achieved by limiting the exposure for sample ablation and by permanent monitoring of the number concentration of the particles in the buffer vessel. Stabilization of the number concentration of the particles is achieved by using the accumulation vessels. For the flow rate of gas under analysis equal to $1 \mathrm{l} / \mathrm{min}$, the characteristic time of concentration change in the vessel is about 30 minutes, which gives a good stabilization effect as the measurement time is 4 minutes. It should also be noted that the diffusion coefficient is independent of the density of particle material. And of course, regular tests are performed to compare the performance of our equipment with that of others and electronic microscopy ${ }^{6)}$.

\section{Results and discussion}

While studying the ablation of inorganic absorbing samples, we discovered an empirical fact that the addition of water to the sample prevents it from agglomerating.

At present, the physical mechanisms of the soft ablation remain not completely clear. One of the assumed physical mechanisms of soft ablation is the absorption of the terahertz radiation by water. Water present between the sample particles strongly absorbs the $\mathrm{THz}$ FEL radiation, which causes the pulsed evaporation of water and brings a part of mac- 
romolecules or clusters of the sample together with water vapor into the gas phase. The effect of water on wablation established empirically becomes more clear from this point of view.

Size distributions for the ultrafind diamond-carbon powder and ultrafine $\mathrm{SiO}_{2}$ powder determined using different measurement procedures are shown in Fig. 3 and Fig. 4. Average sizes of the major (in counted number) fraction of particles and the width of distribution are shown in the figures. For the main numeric fraction, we see a good agreement of the results obtained by means of the soft ablation with the results obtained using scanning electron and atomic force microscopy. Complexes of coarse aggregates of particles (with the mean size of $100 \mathrm{~nm}$ and more), obtained wite soft ablation and dynamic light scattering, are seen in the right-hand parts of the plots. One can also see a low-molecular fraction in Fig. 4 in the left-hand part of the plot; this fraction was successfully recorded only with the help of soft ablation.

In either of the cases, only the aggregated particles were detected with dynamic light scattering in spite of our attempts te break the disaggregates by treating the sample with ultrasound and SDS. Disagreement of the DLS data with data obtained using the other methods results from a specific feature of this method. Thus, intensity of the scattered light is strongly dependent on the molar mass of the sample. Even a small-by-number fraction of large particles overcomes the signal from smaller scatterers and the small size fraction cannot be seen in the presence of a few massive aggregates.

Comparison of the results obtained using four independent methods allows us to hope that we determined the actual fractional composition of the powder sample, even in spite of the difference in the technology of sample preparation. A positive feature of submillimeter laser ablation is the actual absence of sample preparation. In this situation, only the particles of the initial substance can enter the aerosol phase. A low quantum energy of the radiation does not allow one to split the material below the threshold of chemical bonds, which was confirmed previously in the experiments with such «delicate» biological structures as enzymes and DNA ${ }^{4,5}$.

It should be stressed additionally thae investigation of the samples by means of TEM/SEM/DLS involves time- and labor-consuming cycles of sample preparation, measurement and analysis of the results obtained, while a single determination of the fraction

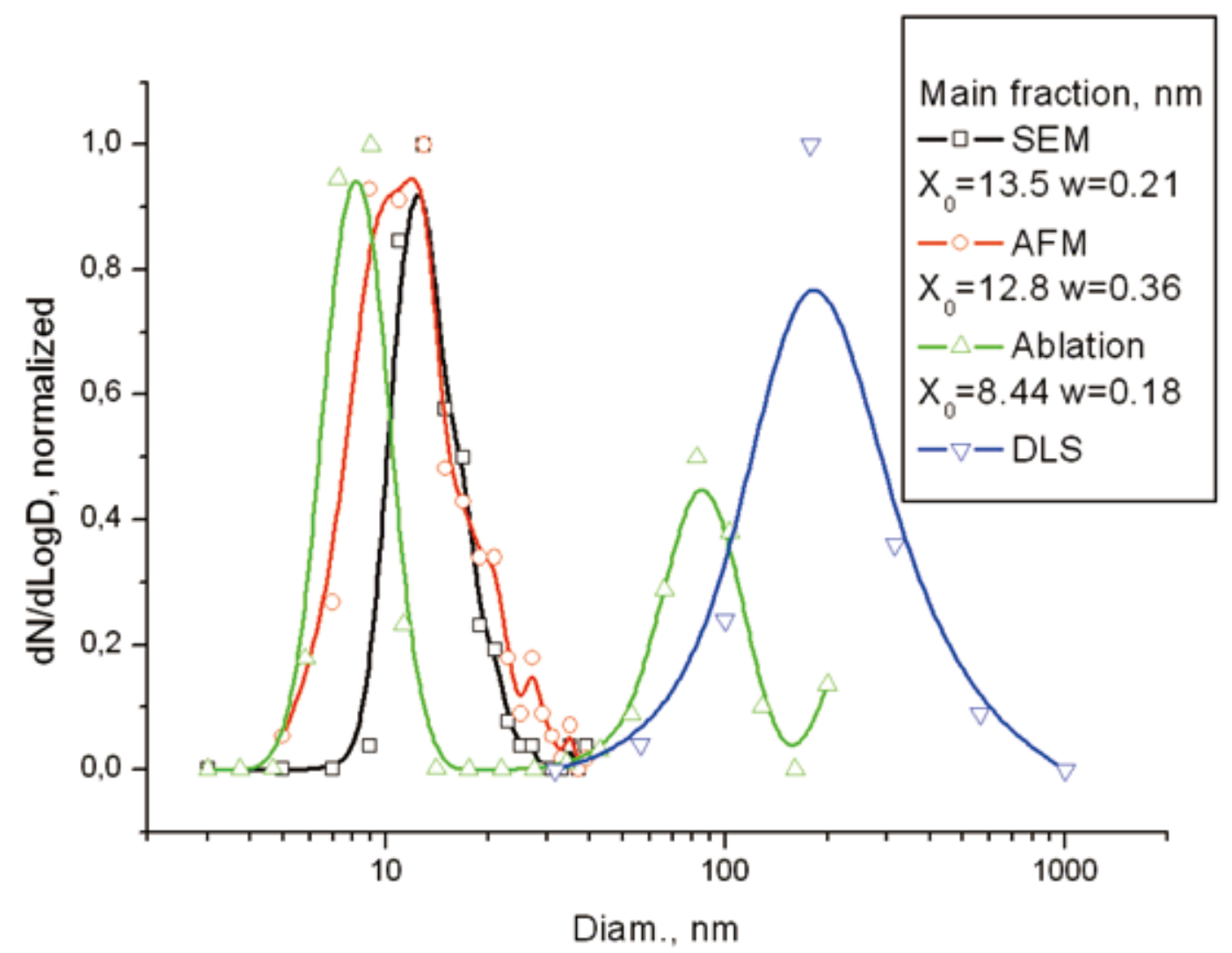

Fig. 3 Size distributions of diamond carbonaceous powder particles registered by scanning electron microscopy (SEM), atomic force microscopy (AFM), dynamic light scattering (DLS) and aerosol products of submillimeter laser ablation (Ablation). 


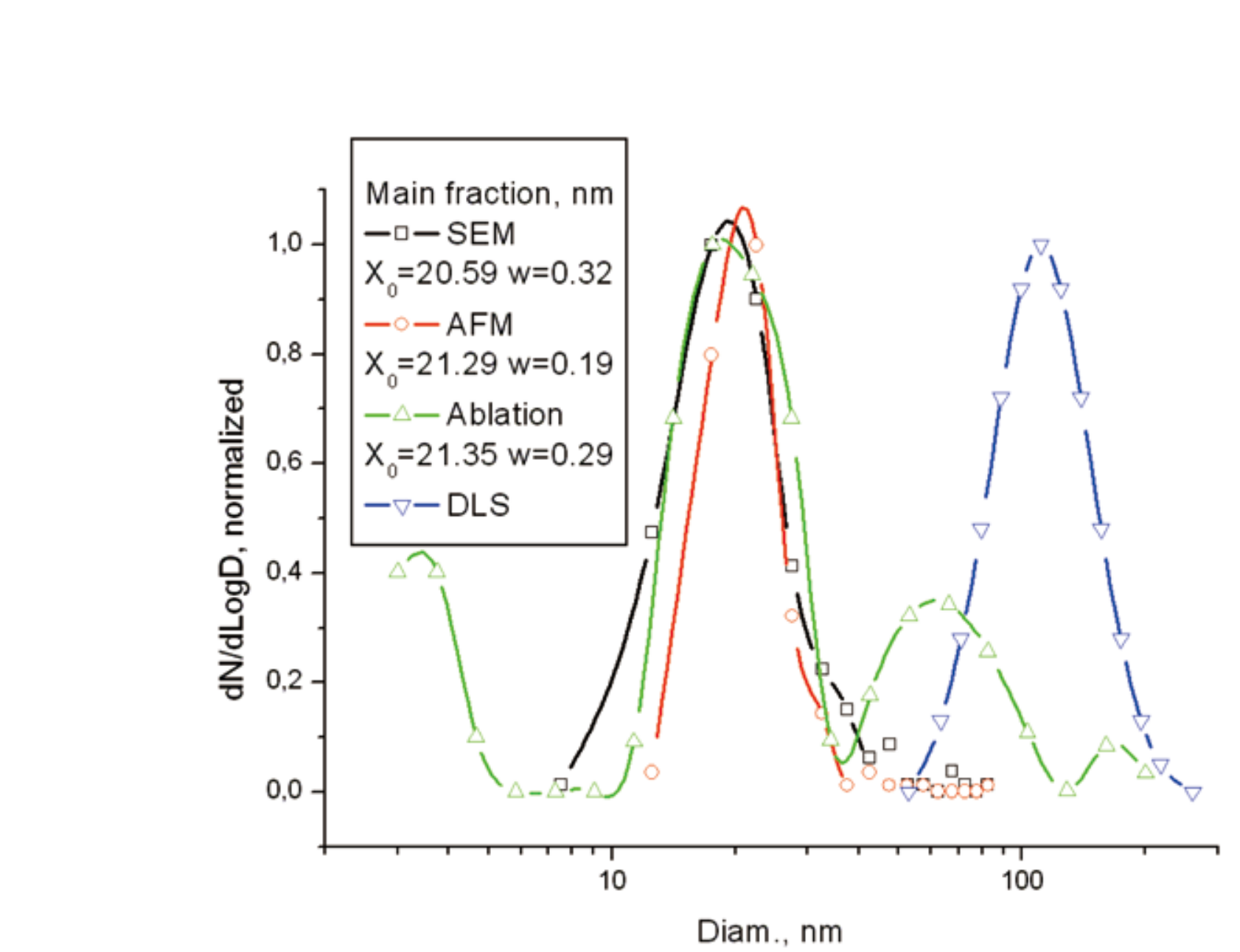

Fig. 4 Size distributions of $\mathrm{SiO}_{2}$ powder particles registered by scanning electron microscopy (SEM), atomic force microscopy (AFM), dynamic light scattering (DLS) and aerosol products of submillimeter laser ablation (Ablation).

composition of these samples by means of analysis of the aerosol products of the submillimeter laser ablation took about 30 minutes. In addition, our method allows one not only to determine the size of particles in a mixture but also to determine the numerical concentrations of the fractions with good accuracy.

\section{Résumé}

The soft nondestructive ablation under the action of the terahertz radiation is an efficient method of transferring macromolecules and clusters from solid substrates and solutions into the aerosol phase while retaining the structural and functional features.

In comparison with conventional methods, the soft ablation method exhibits not only a good agreement of the mean particle size but also turned out to be more informative as it all of particle fractions that are non-recordable using other methods to be detected.

At present, the authors are working on mastering and optimizing the proposed method and plan to broaden the range of samples suitable for this analytical technique. We hope that our approach can become universal, that is, equally applicable in biol- ogy, chemistry of polymers, and in the investigations of organic and inorganic clusters of any origin.

\section{Acknowledgements}

The work was supported by INTAS - SB ant Grant No.: 06-1000013-8569, SB ant Grant No.: 52/20ors The authors thank the researchers from the Chemical Department of the University of Helsinki: Mikko Karesoja, Esa Puukilainen and Matti Elomaa for assistance in the investigation.

\section{List of symbols}

$D \quad$ diffusion coefficient

$K \quad$ penetration coefficient

$k \quad$ Boltzmann constant

$l \quad$ free path length of gas molecules

$N$ number concentration

$n \quad$ number of screens

$r \quad$ radius of particle

$T$ temperature

Greek symbols

$\eta \quad$ gas viscosity 
parije

density of particles distribution count distribution of particles

\section{References}

1) Luk' yanchuk, B. (2002): "Laser Cleaning”, World Scientific, New Jersey.

2) Vogel A., Venugopalan V. Mechanisms of pulsed laser ablation of biological tissues (2003): Chemical Reviews., Vol.103, pp.577-644.

3) Gavrilov, N.G., Knyazev, B.A., Kolobanov, E.I., Kotenkov, V.V., Kubarev, V.V., Kulipanov, G.N., Matveenko, A.N., Medvedev, L.E., Miginsky, S.V., Mironenko, L.A., Oreshkov, A.D., Ovchar, V.K., Popik, V.M., Salikova, T.V., Scheglov, M.A., Serednyakov, S.S., Shevchenko, O.A., Skrinsky, A.N., Tcheskidov, V.G., Vinokurov, N.A. Status of the Novosibirsk high-power terahertz FEL (2007): Nuclear Instruments and Methods in Physics Research A., Vol.575, pp.54-57.

4) Petrov, A.K., Kozlov, A.S., Malyshkin, S.B., Taraban, M.B., Popik, V.M., Scheglov, M.A., Goriachkovskaya, T.N., Peltek, S.E. Nondestructive transfer of complex molecular systems of various origin into aerosol phase by means of submillimeter irradiation of free electron laser (FEL) of Siberian center for photochemical research (2007): Nuclear Instruments and Methods in Physics Research A., Vol.575, pp.68-71.

5) Petrov, A.K., Kozlov, A.S., Taraban, M.B., Goryachkovskaya, T.N., Malyshkin, S.B., Popik, V.M. and Peltek, S.E. Soft ablation of biological objects caused by free-electron laser submillimeter radiation (2005): Doklady Biochemistry and Biophysics., Vol.404, pp.357-359.

6) Ankilov, A., Baklanov, A., Colhoun, M., Enderle, K.H., Gras, J., Julanov, Yu., Kaller, D., Lindner, A., Lushnikov, A.A., Mavliev, R., McGovern, F., O’ Connor, T.C., Podzimek, J., Preining, O., Reischl, G.P., Rudolf, R., Sem, G.J., Szymanski, W.W., Vrtala, A.E., Wagner, P.E., Winklmayr, W. and Zagaynov, V. Particle size dependent response of aerosol counters (2002): Atmospheric Research., Vol.62, pp.209-237.

7) Schärtl, W. (2007): "Light Scattering from Polymer Solutions and Nanoparticle Dispersions”, Springer Verlag, Berlin Heidelberg, Germany.

8) Fuchs, N.A. (1964): "The Mechanics of Aerosols" , Pergamon Press, Oxford, UK. 


\section{wo Alúthor's short biography}

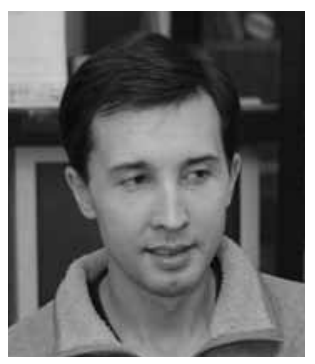

Alexander S. Kozlov

Dr. Kozlov (born 1975) at the present works at the Institute of chemical kinetics and combustion as a researcher and manages the work of a userstation at the Siberian center of photochemical investigation. In 1999 he received the bachelor degree in chemical physics at the Novosibirsk state university. In 2005 he received his Ph.D. in Chemical sciences at the Institute of solid state chemistry and mechanochemistry. His Ph.D. thesis was devoted to the investigation of the various processes of nanoaerosol formation and detection in laboratory and natural conditions. He has more than 20 referred journal publication and over 20 international conference presentations.

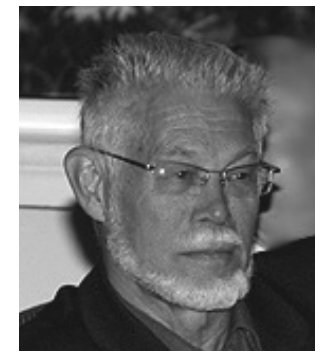

\section{Alexander K. Petrov}

Born 1938

Education: Kemerovo University, Physics, 1961; Institute of Chemical Kinetics and Combustion Novosibirsk, Candidate Thesis, 1967; Institute of Catalysis, Novosibirsk, Doctor Thesis, 1985; Professor, 1994.

Professional: Jr. Researcher, Institute of Organic Chemistry, Novosibirsk 19631971; Institute of Chemical Kinetics and Combustion, Novosibirsk: 1971-1975 - Jr. Researcher; 1975-1986 - Senior Researcher; 1986-1992 - Leading Researcher; 19922008 - Head of Laboratory of Laser Photochemistry; 1993 -2008- Deputy Director of Institute; 1994 - Professor; 2008 - Principal Researcher.

Current Research Interest: Chemical kinetics, Laser Photochemistry, Laser isotope separation, Kinetics of vibration - excited states, Interaction of laser irradiation with matter.

He has over 150 referred publications (including 3 reviews and 2 monographs).

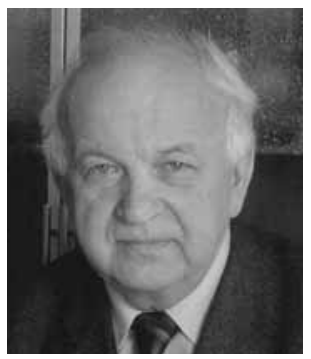

\section{Professor Gennady Kulipanov}

Born 1942.

Education and Career: 1958-1963 Novosibirsk State Technical University; 1963 - Budker Institute of Nuclear Physics; 1970 - Ph.D. in Physics, Budker Institute of Nuclear Physics, SB RAS; 1981 - Head of Laboratory in Budker INP SB RAS; 1991 - Deputy Director of the Budker Institute of Nuclear Physics and Director of Siberian Synchrotron Radiation Center; 1997 - Corresponding Member of Russian Academy of Sciences; 1999 - 2008 Vice-chairmen of Siberian Branch of Russian Academy of Sciences; 2002 - Academician of RAS; 2008 - Deputy Director of the Budker Institute of Nuclear Physics and Director of Siberian Synchrotron and Terahertz Radiation Center.

Research Interests: Physics of nonlinear phenomena in particle accelerators, generation and application of the synchrotron radiation (SR), free electron lasers (FEL), $\mathrm{THz}$ application. He is the author more than 300 publications (including 14 reviews and 1 monograph).

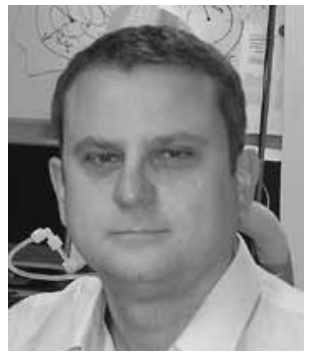

\section{Vladimir O. Aseyev}

Docent Dr. Aseyev (born 1968) is a senior scientist and works as a university lecturer at the Laboratory of Polymer Chemistry, University of Helsinki, Finland. He received his $\mathrm{PhD}$ in chemistry in 1999 at the same Laboratory and became a Doctoral Candidate in Physics and Mathematics in 2000 at the Russian Academy of Sciences, St. Petersburg, Russia. During 1999-2001, he worked at the Department of Chemical and Biochemical Engineering, University of Iowa, USA as a postdoctoral associate and then returned back to Finland. His area of interests includes physics and chemistry of water-soluble and -swellable polymers and nanoparticles. 